\title{
Bioassay Class
}

National Cancer Institute

\section{Source}

National Cancer Institute. Bioassay Class. NCI Thesaurus. Code C42886.

An abstract class which represents both physical and computational groupings of arrays and biomaterials. 